\title{
SVM-Based Biometric Authentication Using Intra-Body Propagation Signals
}

\author{
Isao Nakanishi Yuuta Sodani \\ Graduate School of Engineering, Tottori University \\ 4-101 Koyama-minami, Tottori, 680-8552 Japan \\ nakanishi@ele.tottori-u.ac.jp
}

\begin{abstract}
To use intra-body propagation signals for biometric authentication have been proposed. The intra-body propagation signals are hid in human bodies; therefore, they have tolerability to circumvention using artifacts. Additionally, utilizing the signals in the body enables liveness detection with no additional scheme. The problem is, however, verification performance using the intra-body propagation signal is not so high. In this paper, in order to improve the performance we propose to use user-specific frequency bands for all users in verification. The verification performance is improved to $70 \%$. Furthermore, we introduce the support vector machine (SVM) into the verification process. It is confirmed that verification rate of about $86 \%$ is achieved.
\end{abstract}

\section{Introduction}

Biometrics for user authentication has attracted a great deal of attention. As representative examples, there are a fingerprint, iris, vein, face, voice, and signature and they are used in various applications [1]. They are, however, almost revealed on a body surface or captured outside the body; therefore, it is comparatively easy to forge them using artifacts [2]. Only the vein is hidden in the body but there is a report that authentication systems using the vein accepted artifacts in enrollment and verification [3]. This is due to lack of liveness detection which examines whether an object is a part of a living body. The liveness detection scheme is necessary for protecting the biometric authentication system from deceiving using artifacts while it requires some additional sensor or system.

We have proposed to use intra-body propagation signals as biometrics [4], which are electromagnetic waves propagated at relatively shallow depth in human bodies. The body structure of each individual human being is different from those of others; therefore, it results in some difference in the transfer characteristic of his/her intra-body propagation. Of course, since the intra-body propagation signal is hidden in the body, it is effective for anti-circumvention. In addition, it is possible to realize the liveness detection scheme with no additional sensor.

In [4], the spectrum of the intra-body propagation signal was extracted as a feature vector and the similarity based on the Euclidean distance between the spectrum and its template was evaluated in verification. But the verification rate obtained was $58 \%$ and it was too low.

In this paper, we propose to use user-specific frequency bands for all users in verification since users have different distribution of the spectrum. In addition, in order to improve the performance further, we introduce the support vector machine (SVM) into the verification process.

The present paper is organized as follows: In Sect. 2, the intra-body propagation signal is explained and the verification performance by using the user-specific frequency bands is presented. Next, the SVM is explained and the results by the verification using the SVM are shown in Sect. 3. Finally, the conclusions are presented in Sect. 4.

\section{Intra-body propagation signal}

For obtaining intra-body propagation signals, the technology for intra-body communication was utilized. There have been proposed three transmission modes for the intrabody communication: the simple circuit type, the electrostatic coupling type, and the waveguide type. Particularly, the waveguide type regards a human body as a waveguide and an input signal at an input electrode pair is propagated to an output electrode pair as an electromagnetic wave. In addition, the waveguide type is insensitive to environmental disturbances and can transmit wide frequency band signals.

\subsection{Measurement}

In this paper, for measuring intra-body propagation signals we utilize the waveguide type, which consists of general-purpose measuring instruments: a signal generator, digital oscilloscope as a detector and body surface electrodes with gelled pads. The diagram of connection is shown in Fig. 1. The measurement site is antebrachial region for making the measurement easy. A white noise 


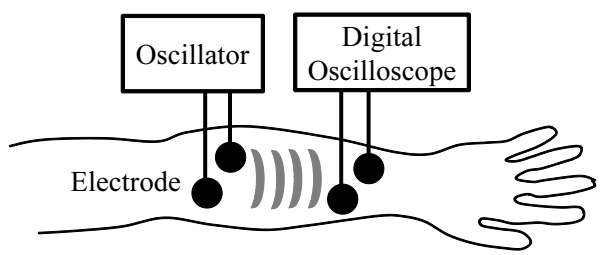

Figure 1. Measurement of intra-body propagation signals.

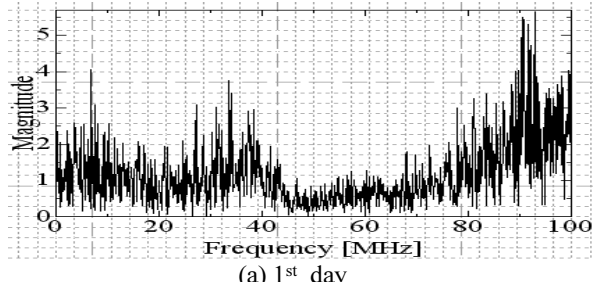

(a) $1^{\text {st }}$ day

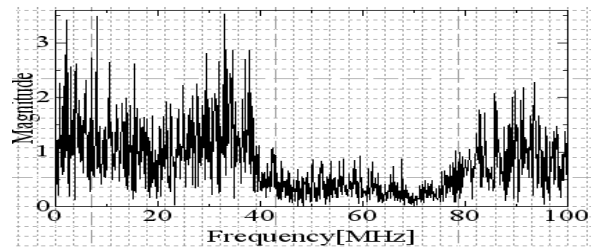

(b) $2^{\text {nd }}$ day

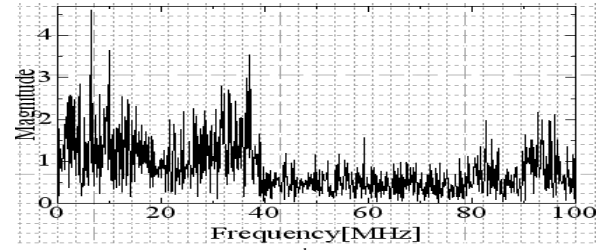

(c) $3^{\text {rd }}$ day

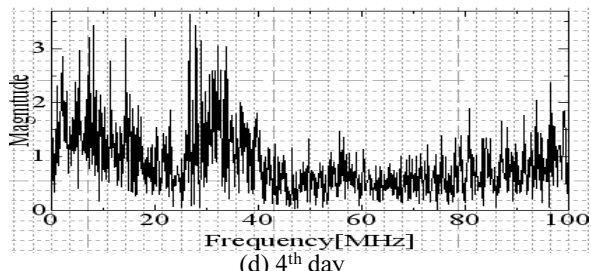

d) $4^{\text {th }}$ day

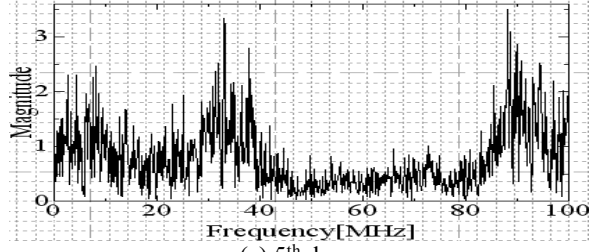

(e) $5^{\text {th }}$ day

Figure 2. Intra-variation of the intra-body propagation spectrum.

signal is passed through one electrode pair from the signal generator. On the other hand, a leaked electromagnetic wave is propagated and then extracted at the digital oscilloscope through another electrode pair. While measuring arms are kept in the air since the amplitude of the propagated signal is reduced when the arms are set on the desk.

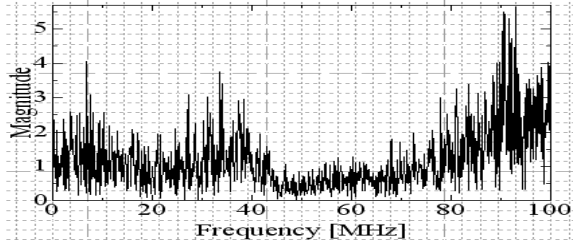

(a) Subject A

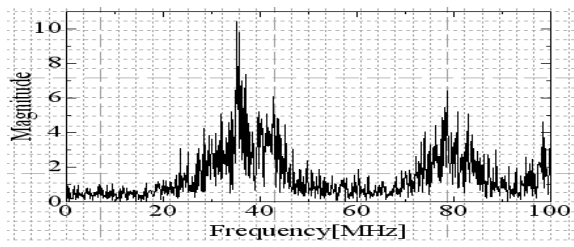

(b) Subject B

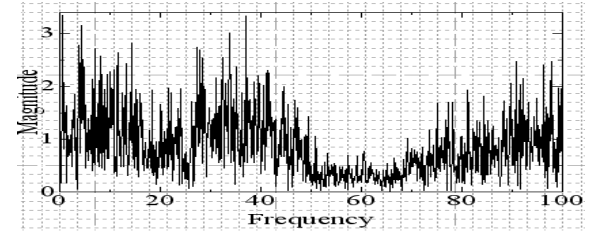

(c) Subject C

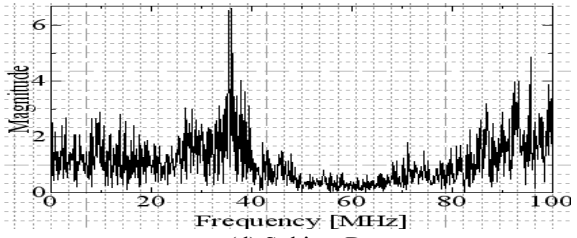

(d) Subject D

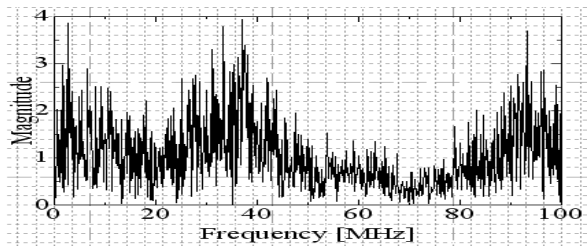

(e) Subject E

Figure 3. Inter-variation of the intra-body propagation spectrum.

\subsection{Feature Extraction}

As individual features, we extract the spectrum of the intra-body propagation signal using the FFT. Figure 2 shows spectra of a subject on five days, that is, intravariation of the intra-body propagation spectrum is observed.

It is confirmed that these spectral distribution have some commonality but partly differ, for example, at $80-100 \mathrm{MHz}$. Therefore, it is anticipated that the intra-variation of the intra-body propagation spectrum is relatively large.

Next, we show the spectra of five subjects (A, B, C, D, and E) in Fig. 3, where inter-variation of the intra-body propagation spectrum is observed.

Detailed observation suggests that individuals have different distribution of the spectrum but for instance, spectra of the subjects: $\mathrm{C}$ and $\mathrm{E}$ are similar at 0-50 MHz; therefore, 


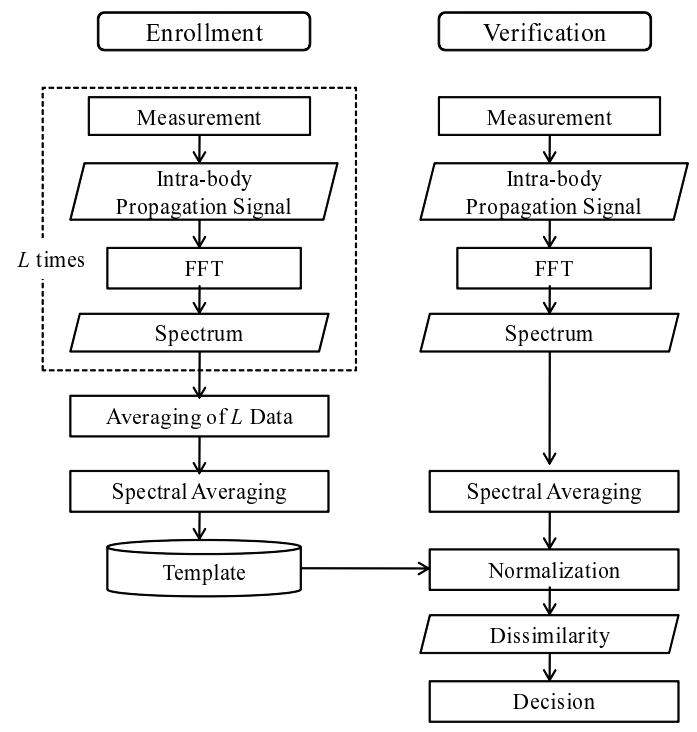

Figure 4. Flow diagram of conventional verification.

it is assumed that the inter-variation is not all that large.

From the above comparisons, the spectrum of the intrabody propagation signal is expected to be as individual features while for that it is necessary to examine which frequency bands should be used for better verification performance since some have large intra-variation, and some have small inter-variation.

\subsection{Verification}

Verification is performed based on the dissimilarity of the intra-body propagation spectrum. The flow diagram of the verification is depicted in Fig. 4.

First of all, templates are prepared as comparison targets in the enrollment stage. An intra-body propagation signal of a user is measured and then an amplitude spectrum is extracted. Repeating it $L$ times and then ensemble-averaging the $L$ amplitude spectra, the template for the user is obtained.

However, the intra-variation of the intra-body propagation spectrum is large. Therefore, smoothing is applied to the spectrum. Concretely, sampled data obtained in a single measurement are divided into several subgroups, a spectrum is extracted in each subgroup, and then spectra in all subgroups are ensemble-averaged. As the number of subgroups is increased, the effect of the smoothing might be elevated but the number of data for FFT analysis is decreased and it results in reduction of frequency resolution and thereby might degrade the verification performance. The smoothed spectrum and its mean value are stored as the template. This procedure is performed for all users in advance to verification.

On the other hand, in the verification stage, each user declares who oneself is by giving his/her name or ID number to the system, which specifies the template. And the intrabody propagation signal for verification (verification signal) is measured and then the smoothed spectrum and its mean value are extracted in the same way as templates.

Next, normalization is performed by equalizing the mean value of the verification spectrum with that of the template, and the dissimilarity is calculated as

$$
\text { Dissimilarity }=1-\frac{\sqrt{\sum_{N}^{M}\left(t_{k}-v_{k}\right)^{2}}}{\sqrt{\sum_{N}^{M} t_{k}^{2}}+\sqrt{\sum_{N}^{M} v_{k}^{2}}}
$$

where $t_{k}, v_{k}$ are the amplitude spectrum of the verification signal and the template, respectively. $k$ is a frequency index, and $M$ and $N$ are respectively upper and lower limits of the frequency band used in the verification.

The numerator of the second term in the right-hand side corresponds to Euclidian distance between the verification spectrum and the template. The denominator is their power spectra; therefore, the distance is normalized and as a result the dissimilarity always falls within 0 and 1 .

Finally, the dissimilarity is compared with a threshold. If the dissimilarity is smaller than the threshold, the user presented the verification signal is regarded as genuine.

\subsection{Experiments}

We had carried out the experiments using 20 subjects in order to evaluate the ability of the person authentication using the intra-body propagation signal. The measurement was done ten times on a day for each subject and it was repeated five times (days). Total number of data was 1000 . The number of data for making the template $L=5$ was five. Concretely, five data was randomly chosen from ten data measured on the first day and then they were averaged at each frequency bin.

The frequency band of a white noise was $0-100 \mathrm{MHz}$ and its amplitude was $2.0 V_{p-p}$. Sampling frequency was 500 $\mathrm{MHz}$ and the number of sampled data was 4000; therefore, measurement time was $8 \mu \mathrm{s}$.

Verification performance was evaluated by using the Equal Error Rate (EER) at which the False Rejection rate (FRR) is equal to the False Acceptance Rate (FAR). First, we show the EERs according to the variation of the average number for smoothing in Table 1 where whole frequency band $(0-100 \mathrm{MHz})$ was used in the verification.

It is confirmed that the EER was hardly improved even if the average number was increased. The reason is that frequency resolution was inversely degraded as the average number was increased. In the following, the average number was set to 8 . 
Table 1. EERs according to the variation of the average number for smoothing.

\begin{tabular}{|c||c|c|c|c|}
\hline Average number & 0 & 8 & 10 & 20 \\
\hline EER (\%) & 56 & 39 & 39 & 40 \\
\hline
\end{tabular}

Table 2. EERs at sub-bands.

\begin{tabular}{|c|c|}
\hline Band $(\mathrm{MHz})$ & EER $(\%)$ \\
\hline $0-10$ & 44 \\
\hline $10-20$ & 43 \\
\hline $20-30$ & 41 \\
\hline $30-40$ & 47 \\
\hline $40-50$ & 42 \\
\hline $50-60$ & 35 \\
\hline $60-70$ & 37 \\
\hline $70-80$ & 38 \\
\hline $80-90$ & 50 \\
\hline $90-100$ & 56 \\
\hline
\end{tabular}

Next, we divided the frequency region: 0-100 MHz into ten sub-bands and calculated an EER at each band. The results are summarized in Table 2.

Every sub-band had a quite different EER. In particular, the smallest EER of $35 \%$ was achieved at 50-60 MHz. Resultingly, it is confirmed that the verification performance depends on the frequency band used in verification. This result agrees with the consideration about the intra- and intervariation of the intra-body propagation spectrum in Sect. 2.2 .

As a result, we calculated EERs at all sub-bands for every subject. A part of the results is summarized in Table 3, where the smallest EER and the sub-band for obtaining it are shown for all subjects (No. 1-20). If plural sub-bands had the smallest EER, the nearest one to 50-60 MHz was chosen.

It is found that the sub-band of $50-60 \mathrm{MHz}$ is not suitable for all subjects. In other words, every subject has the most appropriate sub-band for verification. So assuming that such the appropriate sub-band is used for each subject, we calculated total EER and as a result we obtained the EER of $30 \%$ while the EER was $42 \%$ in [4].

At the present time there is no criterion to determine which sub-band is the most suitable for every subject; therefore it have to be decided by the EER using a number of subjects' data. In practical applications, it is not engaged that such enough data are enrolled in authentication systems. How to seek discriminative frequency bands for users is a problem to be studied.
Table 3. The smallest EER and its sub-band for all subjects.

\begin{tabular}{|c|c|c|}
\hline Subject No. & EER (\%) & Band (MHz) \\
\hline \hline 1 & 15 & $50-60$ \\
\hline 2 & 17 & $70-80$ \\
\hline 3 & 18 & $50-60$ \\
\hline 4 & 28 & $50-60$ \\
\hline 5 & 37 & $40-50$ \\
\hline 6 & 36 & $50-60$ \\
\hline 7 & 27 & $70-80$ \\
\hline 8 & 2 & $0-10$ \\
\hline 9 & 33 & $50-60$ \\
\hline 10 & 31 & $40-50$ \\
\hline 11 & 28 & $50-60$ \\
\hline 12 & 43 & $30-40$ \\
\hline 13 & 37 & $50-60$ \\
\hline 14 & 36 & $30-40$ \\
\hline 15 & 33 & $60-70$ \\
\hline 16 & 29 & $70-80$ \\
\hline 17 & 35 & $70-80$ \\
\hline 18 & 37 & $20-30$ \\
\hline 19 & 32 & $70-80$ \\
\hline 20 & 16 & $70-80$ \\
\hline & & \\
\hline
\end{tabular}

\section{Introduction of SVM into Verification}

As described above, the verification based on the Euclidian distance is simple but not adequate for employing the authentication using the intra-body propagation signal in practical applications, so that we introduce the support vector machine (SVM) into the verification process in order to improve the verification performance further.

\subsection{SVM}

As a pattern classification method based on supervised learning, the SVM has been proposed [5]. The SVM learns a separating hyperplane which maximizes the distance (margin) between two classes and so performs higher separation capability for unlearned data of the classes

Figure 5 shows an illustration of the separating hyperplane of two classes: $\mathrm{C}_{1}$ and $\mathrm{C}_{2}$. However, the two classes in practical applications are almost linearlyinseparable. Therefore, they are transformed to higherdimensional space by using a kernel function and thereby become linearly-separable there. In general, the polynomial kernel, Gaussian kernel, and RBF kernel are used as the kernel function.

\subsection{Verification Using SVM}

The flowchart of verification with the SVM is shown in Fig. 6. In advance to the verification, the enrollment (learn- 


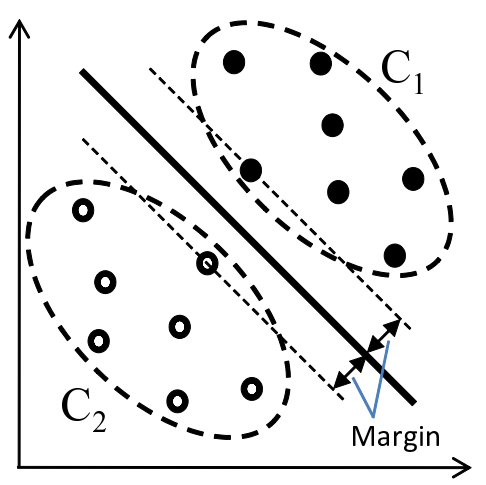

Figure 5. A separating hyperplane between two classes.

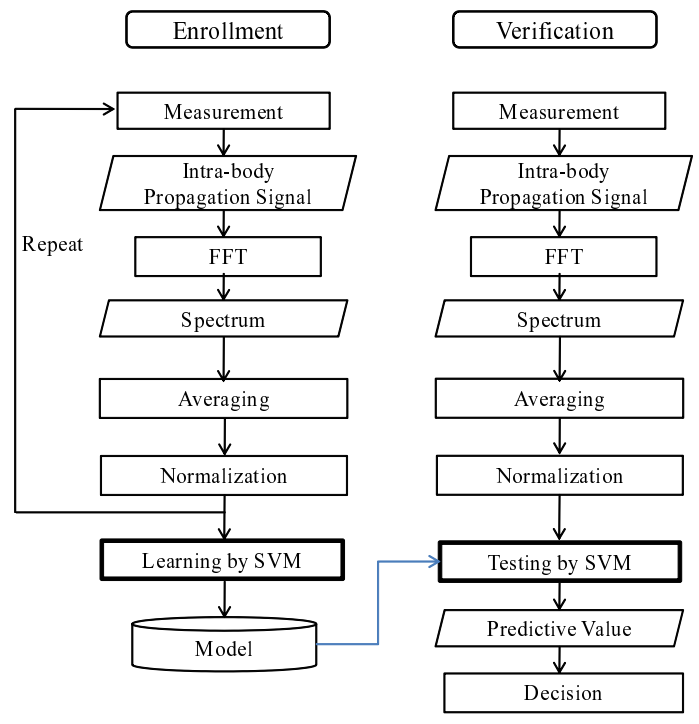

Figure 6. Flowchart of verification with the SVM.

ing) phase is performed. For preparing learning data, intrabody propagation signals for all users are measured and then their spectra are extracted and smoothed and normalized. The smoothing is the same as the conventional method. In the normalization, mean values of all amplitude spectra are adjusted to one. User specific models are constructed by supervising the classification of the user's own data and others' ones to +1 and -1 , respectively.

In the verification (testing) phase, a spectrum of a user is input to the corresponding model, that is, already-learned SVM and then the SVM output a predictive value. If the value has positive sign, the spectrum is regarded as of the genuine user. Inversely, if the value is negative, the user is regarded as an imposter.

\subsection{Experiments}

We used SVM ${ }^{\text {light }}[6]$ with the one-dimensional polynomial kernel.

Intra-body propagation signals obtained from 20 sub- jects in Sect. 2.4 were used and smoothed spectral data of 100 dimensions were used as individual features, again. 40 data from each subject and $20 \times 19$ from others were used in the learning of individual models and 10 data from each subject and $10 \times 19$ from others were used in the verification.

The verification performance was evaluated by using the correct acceptance rate (CAR), correct rejection rate (CRR) and verification rate (VR). The CAR and CJR are the ratio of correctly accepted data and all subjects' own ones $(10 \times 20)$ and that of correctly rejected data and all others' ones $(10 \times 19 \times 20)$, respectively. The verification rate is the ratio of correctly accepted and rejected data and all verification data $(10 \times 20 \times 20)$. The results are presented in Table 4 where the cost parameter: $C$ of the SVM was optimized for each subject.

Table 4. Individual verification performance with optimal cost parameters.

\begin{tabular}{|c|c|c|c|c|}
\hline Subject & $C$ & CAR (\%) & CRR (\%) & VR (\%) \\
\hline \hline A & 1.0 & 80 & 59.5 & 60.5 \\
\hline B & 0.25 & 40 & 82.6 & 80.5 \\
\hline C & 7.0 & 40 & 88.9 & 86.5 \\
\hline D & 1.0 & 100 & 100 & 100 \\
\hline E & 0.05 & 70 & 83.2 & 82.5 \\
\hline F & 10.0 & 10 & 88.9 & 85.0 \\
\hline G & 1.0 & 0 & 100 & 95.0 \\
\hline H & 0.15 & 40 & 85.3 & 83.0 \\
\hline I & 3.0 & 90 & 67.9 & 69.0 \\
\hline J & 0.5 & 50 & 92.6 & 90.5 \\
\hline K & 5.3 & 20 & 84.7 & 81.5 \\
\hline L & 0.55 & 30 & 90.5 & 87.5 \\
\hline M & 1.0 & 70 & 63.7 & 64.0 \\
\hline N & 0.2 & 10 & 96.3 & 92.0 \\
\hline O & 6.0 & 30 & 76.8 & 74.5 \\
\hline P & 1.0 & 80 & 90.5 & 90.0 \\
\hline Q & 0.45 & 60 & 87.4 & 86.0 \\
\hline R & 0.25 & 80 & 88.4 & 88.0 \\
\hline S & 0.7 & 60 & 83.7 & 82.5 \\
\hline T & 0.5 & 20 & 91.1 & 87.5 \\
\hline Ave. & & 49 & 85.1 & 83.3 \\
\hline
\end{tabular}

Totally, the mean VR of $83.3 \%$ was achieved. However, the VR of $100 \%$ was achieved for the subject D while the subject $\mathrm{G}$ was not completely accepted.

To optimize the cost parameter for each user requires excessive computation time in the learning; therefore, it is not appropriate in practical applications. Thus, we examined the verification performance in the condition that all subjects used a common cost parameter. The results are shown in Table 5. The highest VR was about $86 \%$ at $C=0.1$. 
Table 5. Verification performance with common cost parameters.

\begin{tabular}{|c||c|c|c|c|}
\hline$C$ & 0.1 & 0.5 & 1.0 & 5.0 \\
\hline CAR (\%) & 32.5 & 40.5 & 41.5 & 43.0 \\
\hline CRR (\%) & 88.2 & 82.3 & 81.1 & 79.7 \\
\hline VR (\%) & 85.5 & 80.2 & 79.1 & 77.9 \\
\hline
\end{tabular}

On the whole, the CAR was low even when the cost parameter was varied. This is also due to the fact that a subject was completely rejected as confirmed in the previous experiment.

In the learning, the number of each subject' own data was less than those of others, so that the area of his/her class was narrow and might be overlapped by others' one and it yielded inseparable area between the two classes even by using the kernel function. For suppressing such overlapping of the classes, it is a problem to select learning data. Additionally, to use phase spectra as individual features is another solution for improving the verification performance.

\section{Conclusions}

In the biometric authentication based on intra-body propagation signals, we proposed to use user specific sub-bands in verification. The EER of $30 \%$ was obtained and it was roughly equal to verification rate of $70 \%$. Furthermore, in order to improve the performance we introduced the SVM into the verification process. As a result, the verification rate of about $86 \%$ was achieved. There, however, remain problems for improving the performance further.

Our aim is to use the intra-body propagation signal in on-demand authentication of users who are griping cellular phones, mouse devices, or vehicle handles; therefore, we will have to examine intra-body propagation signals in palms.

\section{Acknowledgements}

A part of this work was supported by the Support Center for Advanced Telecommunications Technology Research, Foundation (SCAT) in Japan.

\section{References}

[1] A. Jain, R. Bolle and S. Pankanti, BIOMETRICS Personal Identification in Networked Society, Kluwer Academic Publishers, Massachusetts, USA, 1999. 1

[2] T. Matsumoto, H. Matsumoto, K. Yamada and S. Hoshino, "Impact of Artificial "Gummy" Fingers on Fingerprint Systems," Proc. of SPIE, vol. 4677, pp. 275-289, Jan. 2002. 1
[3] T. Matsumoto, "Security Design and Security Measurement for Biometric Systems (in Japanese)," Proc. of the 7th IEICE Technical Report of Biometrics Security Group, pp. 57-64, 2006. 1

[4] Isao Nakanishi, Yasuhiro Yorikane, Yoshio Itoh, and Yutaka Fukui, "Biometric Identity Verification Using Intra-Body Propagation Signal," Proc. of 2007 Biometrics Symposium, Sep. 2007. 1, 4

[5] N. Cristianini and J. Shawe-Taylor, An Introduction to Support Vector Machines and other kernel-based learning methods, Cambridge University Press, 20004

[6] http://svmlight.joachims.org/ 5 